\section{Collaborative neuroscience}

Working together for a common goal propels neuroscience research.

Collaborations between individual labs are commonplace across many disciplines, but large-scale consortia are perhaps better known in the physics community or in genomics. Yet, in recent years, large-scale initiatives have entered the neuroscience scene.

The BRAIN Initiative Cell Census Consortium and now the BRAIN Initiative Cell Census Network are prime examples of top-down approaches (Neuron 96, 542-557, 2017). Following the announcement of the BRAIN Initiative in 2013, a working group developed the strategic vision; this resulted in funding mechanisms for projects aimed at generating a comprehensive census of cell types in the brains of species ranging from fish and mouse to human. Groups involved in these efforts benchmarked existing approaches and developed new methods in support of this goal, then deployed these methods to generate transcriptomic, epigenomic, anatomical and functional data. Furthermore, they established infrastructure for disseminating and sharing the data, results and resources generated, such that both the community and the public can profit.

In contrast, the International Brain Laboratory (IBL) has emerged from

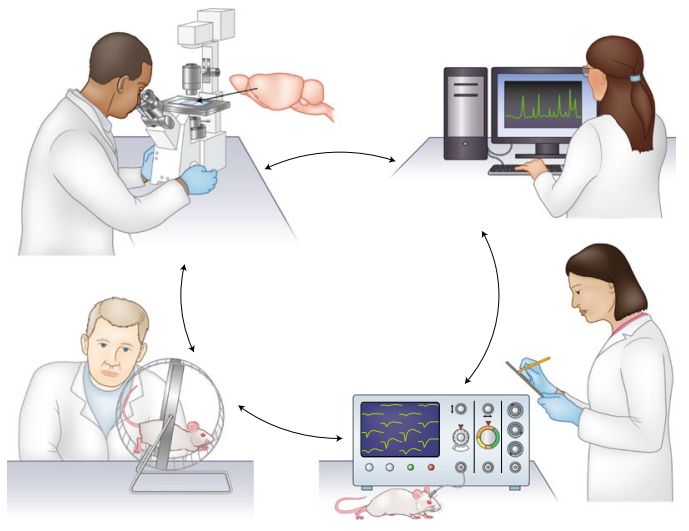

In large-scale collaborations, scientists with different expertise contribute to the project. Credit: Debbie Maizels/Springer Nature

the bottom up (Neuron 96, 1213-1218, 2017). A group of 21 labs with expertise in electrophysiology, calcium imaging, mouse behavior and data analysis teamed up to tackle a common question. They established a data sharing and storage infrastructure and agreed to focus on a visual decision-making task in mouse. To compare data across different labs and to increase reproducibility, the group had to standardize all aspects of this behavioral task before the different labs could embark on recording from different brain areas. The generated data are made available to the whole group for further analysis, and the IBL plans to release them 12 months after generation or upon publication.

Large-scale initiatives like these generate a wealth of data, resources and tools, and making these available to the community is bound to multiply their impact. We look forward to these efforts bearing fruit.

Nina Vogt

Published online: 6 January 2020

https://doi.org/10.1038/s41592-019-0706-2

\section{Exploring microbial worlds} Culturing methods in combination with sequencing technology will help us discover and characterize bacteria.

The continuing development of sequencing technologies has shifted our understanding of the microbial world. Our knowledge, however, is still limited because we cannot isolate and culture the vast majority of microorganisms. Genomicsbased sequencing tools have great advantages for profiling the composition of environmental microbial samples via taxonomic assignment, enabling new species discovery and highlighting the diversity of microbial communities. Yet precise taxonomic assignment is not easy. Computational tools have been developed to aid such metagenomics analysis for better assembly of sequence reads into functional and taxonomic compositions (Nat. Methods 16, 603-606, 2019).

Notably, sequencing-enabled taxonomic compositions heavily depend on the abundance of species in the microbiota, which likely favors dominant populations and thus limits our view of minority

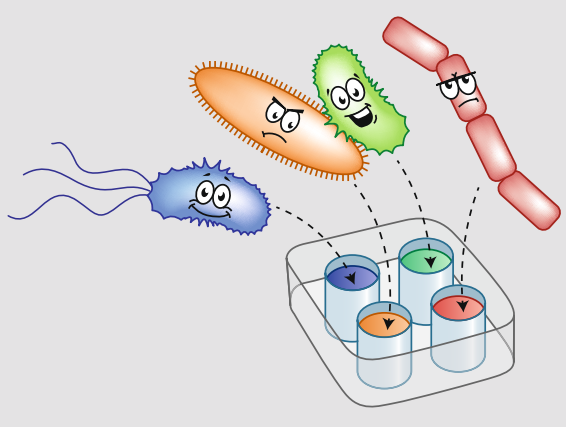

Culturing methods and sequencing technology facilitate the culture of more species.

Credit: Katherine Vicari/Springer Nature

populations. Microbiologists have hence started to revisit culture-dependent methods for previously unculturable microorganisms.

Unknown parameters in growth conditions, such as a requirement for exotic nutrients or interspecies interaction, make culturing a real challenge. But culture-dependent methods beyond conventional techniques are emerging to counteract recalcitrance to cultivation. For example, microfluidics-enabled in-droplet cultivation offers a high- throughput approach to exploring pairwise interactions between signaling cells (Proc. Natl Acad. Sci. USA 114, 2550-2555, 2017).

Targeted culturing of bacteria with specific phenotypes has been developed for culturing a subset of intestinal bacteria (Nature 533, 543-546, 2016). Recently, a genome-informed approach was established to physically isolate microorganisms based on the expression of a membrane protein (Nat. Biotechnol. 37, 1314-1321, 2019).

Surely, sequencing technology will continue to grow and assist microbial profiling, as well as deciphering of the underlying metabolic pathways. Culture-dependent methods in return will afford a way to experimentally validate hypotheses generated from metagenomics studies. We envision microbial cultures and omics technologies serving as complementary tools to broaden our knowledge of bacterial communities.

Lei Tang

Published online: 6 January 2020

https://doi.org/10.1038/s41592-019-0707-1 